\title{
Temporal Correlation between Maximum Tetanic Force and Cell Death in Postischemic Rat Skeletal Muscle
}

\author{
Hidekazu Suzuki, David C. Poole, * Benjamin W. Zweifach, and Geert W. Schmid-Schönbein \\ Department of Bioengineering, Institute for Biomedical Engineering and Department of Medicine, Division of Physiology,* University of \\ California San Diego, La Jolla, California $92093-0412$
}

\begin{abstract}
To gain insight into the mechanisms responsible for muscle dysfunction after ischemia-reperfusion, a rat spinotrapezius muscle preparation was developed which enabled sequential measurements of in vivo maximum tetanic force production and cell death assessed using digital microfluorographic determination of propidium iodide (PI) staining. After $60 \mathrm{~min}$ of no-flow ischemia, maximum tetanic force fell significantly during $90 \mathrm{~min}$ of reperfusion compared with control, nonischemic muscles. The most striking fall was evident within 30 min of reperfusion and occurred concomitant with an explosive increase in PI-positive myocyte nuclei. Treatment with the oxygen radical scavenger, dimethylthiourea, attenuated both the fall in force and increased PI staining. Indeed, the rise in PI-positive nuclei correlated closely $(r=0.728)$ with the reduction of maximum tetanic force developed following ischemia and reperfusion under all conditions. Superoxide dismutase also attenuated the rise in PI-positive nuclei. Assessment of mitochondrial inner membrane potential $(\Delta \psi)$ using Rhodamine 123 fluorescence revealed that myocytes with the lowest initial mitochondrial membrane potential were subject to the greatest injury after $90 \mathrm{~min}$ of reperfusion $(r=0.828)$. These results support the hypothesis that myocyte injury, as visualized by PI-staining, reflects an impaired contractile function in fibers with a low oxidative potential which is likely mediated by oxygen radicals. (J. Clin. Invest. 1995. 96:2892-2897.) Key words: ischemia-reperfusion • oxygen radicals • spinotrapezius muscle $\bullet$ mitochondria $\bullet$ dimethylthiourea
\end{abstract}

\section{Introduction}

Skeletal muscle ischemia is an intrinsic aspect of many acute (e.g., circulatory shock, traumatic injury, surgical intervention) and chronic (e.g., diabetes mellitus, obstructive vascular dis-

Preliminary reports of this work were presented at the 67th Scientific Sessions of the American Heart Association, 15 November 1994, in Dallas, TX.

Address correspondence to Geert W. Schmid-Schönbein, Institute for Biomedical Engineering, University of California, San Diego, 9500 Gilman Drive, La Jolla, CA 92093-0412. Phone: 619-534-3852; FAX: 619-534-5722.

Received for publication 6 March 1995 and accepted in revised form 7 August 1995.

J. Clin. Invest.

(c) The American Society for Clinical Investigation, Inc.

0021-9738/95/12/2892/06 \$2.00

Volume 96, December 1995, 2892-2897 ease) clinical entities. The postischemic period after reperfusion is characterized by a cascade of pathological events. These include microvascular damage (1-3), lipid peroxidation (4), and destruction of sarcolemmal, myofibrillar, and mitochondrial elements $(5,6)$ which preferentially impact fast-twitch glycolytic fibers (7-9). This results in myocyte dysfunction and death thereby impairing overall muscle contractile performance ( 8 , $10,11)$.

There is compelling evidence that oxygen radicals play a major role in muscle ischemia-reperfusion (I/R) ${ }^{1}$ injury $(2,3$, 9) which occurs within minutes to days of an I/R episode (7, $11,12)$. Recently, using an intravital microscopy preparation of the rat spinotrapezius, we were able to demonstrate that myocyte injury (assessed by propidium iodide [PI] staining of nuclei) $(9,13)$ was negligible during a 60 -min period of hypotensive ischemia but increased rapidly (i.e., within $30 \mathrm{~min}$ ) after reperfusion (9). Early myocyte nuclear injury which appeared predominantly in the pericapillary region of fast glycolytic (type IIB) fibers, was attenuated by the inhibition of xanthine oxidase. These events occurred primarily in reperfused capillaries before substantial leukocyte accumulation. Such observations support the notion that oxygen radicals generated within the capillary endothelium might contribute to or initiate the observed reperfusion injury (9).

The consummate test of the contractile integrity of a muscle is its ability to develop force. Indeed, after acute I/R trauma, muscle functional deficits will have a negative impact on the mobility of the individual and their ultimate recovery. To date, it is not known whether the early manifestations of $I / R$ injury described above result in immediate functional deficits. This investigation utilized a novel preparation which combined intravital structural and functional determination of muscle integrity and was designed to test the following hypotheses: $(a)$ muscle contractile function is impaired during the immediate postischemic period and is correlated temporally with increased PI staining; (b) reperfusion injury occurs predominantly in those myocytes with low mitochondrial volumes (assessed by Rhodamine 123 fluorescence); (c) application of the exogenous hydroxyl radical scavenger, dimethylthiourea (DMTU), will reduce myocyte injury and enhance muscle contractile performance during the immediate postischemia period; and $(d)$ application of SOD will reduce myocyte injury during the postischemic period.

\section{Methods}

All animal procedures were reviewed and approved by the University of California San Diego Animal Subject Committee. Male Wistar rats

1. Abbreviations used in this paper: $\mathrm{CCD}$, charge-coupled device; DMTU, dimethylthiourea; GPX, glutathione peroxidase; I/R, ischemiareperfusion; PI, propidium iodide; Rh123, Rhodamine 123. 
(250-350 grams, Charles River Laboratories, Inc., Wilmington, MA) were housed in a controlled environment and maintained on a standard pellet diet for at least $4 \mathrm{~d}$ before initiation of the experimental procedures. One femoral artery and vein were cannulated under general anesthesia (pentobarbital sodium, $40 \mathrm{mg} / \mathrm{kg}$ i.m.).

Muscle preparation. The spinotrapezius muscle is a thin strap-like muscle extending along the spine in the thoracic and upper lumbar region of rodents. The muscle fibers arise from the spines of the fourth thoracic to the third lumbar vertebrae and converge to their insertion on the scapular spine (14). The spinotrapezius muscle was prepared without disturbing its fascia to minimize surgical tissue damage and its associated microcirculatory consequences, leaving the origin and all vascular and nervous connections intact $(14,15)$. The microcirculation was observed via intravital microscopy according to methods described previously $(14,15)$. Briefly, the preparation was placed on a heating pad $\left(37^{\circ} \mathrm{C}\right)$ and was continuously superfused $(2.0 \mathrm{ml} / \mathrm{min})$ with KrebsHenseleit bicarbonate-buffered solution saturated with a $95 \% \mathrm{~N}_{2} / 5 \%$ $\mathrm{CO}_{2}$ gas mixture. The surface of the exposed surrounding tissue was covered with Saran Wrap (Dow Brands L.P., Indianapolis, IN). The tissue was viewed with a water-immersion lens ( $\times 25$ numerical aperture $=0.60$, Leitz, Wetzlar, Germany).

Intravital microscopy and fluorescence measurements. Details of the spinotrapezius muscle microcirculation were documented with an intravital microscope via a digital color charge-coupled device (CCD) camera (DEI-470; Optronics Engineering, Goleta, CA). A single microscopic field $(240 \mu \mathrm{m} \times 330 \mu \mathrm{m})$ containing six to eight muscle fibers with their associated parallel capillaries, and distal venules (and/or proximal arcade arterioles) was initially chosen, followed by observations of adjacent fields ( 6 to 7 fields per muscle). To elicit fluorescent images, the preparation was illuminated with a $200-\mathrm{W}$ mercury lamp. The light was passed through a quartz collector, heat filter (KG-2; Zeiss, Oberkochen, Germany), and fluorescent filter set (Leitz, Ploempak, Germany, excitation filter at $530 \mathrm{~nm}$, barrier filter at $570 \mathrm{~nm}$ ) for visualization of PI fluorescence. PI (Sigma Chemical Co., St. Louis, MO) was added to the superfusion buffer at a final concentration of $1.0 \mu \mathrm{M}$, $20 \mathrm{~min}$ before the in vivo measurements. Staining with PI has been shown to serve as a suitable marker for irreversible nuclear damage $(9,16)$. To minimize photobleaching, the total exposure times of PI fluorography for a single measurement was limited to $<20 \mathrm{~s}$ by using a shutter between the light source and a filter cube. Fluorescence images were videotaped (model AG-1270P; Panasonic Corp., Tokyo, Japan) and randomly selected images in the capillary network of skeletal muscle were stored in a digital processor (Macintosh Laboratory Computer II Ci; Apple Computer Co., Cupertino, CA). In each muscle six to seven fields were selected.

Mitochondrial membrane potential in each muscle fiber was investigated by estimating Rhodamine 123 ([Rh123]; Sigma Chemical Co.) fluorescence intensity (\%Rh) (13). Rh123 is a fluorescent dye that accumulates elecrophoretically in the mitochondria of viable cells in response to a potential difference $(\Delta \psi)$ across the mitochondrial inner membrane (17). Rh123 was dissolved in the Krebs-Henseleit superfusate to a final concentration of $0.8 \mu \mathrm{M}$, which is low enough to maintain mitochondrial viability (18). The spinotrapezius muscle was superfused with the Rh123-containing Krebs-Henseleit solution for $40 \mathrm{~min}$. The $\mathrm{Rh} 123$ was then rinsed away by superfusion with Rh123-free KrebsHenseleit solution for $10 \mathrm{~min}$. To elicit fluorescent images, the preparation was illuminated with a $200-\mathrm{W}$ mercury lamp. The light was passed through a quartz collector, heat filter and excitation filter for epiillumination of the preparation. Rh123-associated fluorescence emission was passed through a 515-nm band pass filter (Leitz). Rh123-derived fluorographs were visualized intermittently using a digital color CCD camera. During each fluorescent light reading the digital controls of the color CCD camera were set at constant camera sensitivity and shutter speed ( contrast $=0$, brightness $=0$, manual integration $=1 / 8$ ) so that the camera served as a light intensity indicator.

Photobleaching of these fluorescent images was minimized by limiting light exposure of the tissue to $<1 \mathrm{~s}$ with a shutter. During the intervening periods, the shutter for the excitation light was kept closed.
The images were recorded with a videocassette recorder for playback analysis. Fluorescence images of the spinotrapezius muscle fibers were digitized $(512 \times 512,8$-bit deep, Image 1.35 with a Macintosh IIci computer) and stored on a removable hard disk for subsequent analysis.

Since the mitochondrial inner membrane potential $(\Delta \psi)$ is proportional to the logarithmic values of the intensity of $\mathrm{Rh} 123$ fluorescence, the relative depletion of membrane potential $(\Delta \psi)$ in each muscle fiber could be estimated according to the following equation, as described elsewhere $(13,16)$ :

$$
\begin{aligned}
\Delta \psi(\mathrm{x}, \mathrm{y}) & =\Delta \psi(\mathrm{x}, \mathrm{y})-\Delta \psi\left(\mathrm{x}_{1}, \mathrm{y}_{1}\right) \\
& =K \log \left\{\mathrm{I}_{\mathrm{Rh}}(\mathrm{x}, \mathrm{y})\right\}-K \log \left\{\mathrm{I}_{\mathrm{Rh}}\left(\mathrm{x}_{1}, \mathrm{y}_{1}\right)\right\} \\
& =K \log \left\{\mathrm{I}_{\mathrm{Rh}}(\mathrm{x}, \mathrm{y}) / \mathrm{I}_{\mathrm{Rh}}\left(\mathrm{x}_{1}, \mathrm{y}_{1}\right)\right\} \\
& =K \log (\% \mathrm{Rh} / 100)(\mathrm{mV})
\end{aligned}
$$

where $K$ is the constant obtained using the Nernst equation $(13,17)$. $I_{R h}(x, y)$ is the intensity at pixel $(x, y)$ in Rh123-associated fluorographs. $I_{R h}\left(x_{1}, y_{1}\right)$ is the maximum Rh123-associated fluorescence intensity in the observation field. In this experiment, the value of $\Delta \psi(t) /$ $\mathrm{K}$ was computed as $\% \mathrm{Rh}$ in each muscle fiber.

During 60-min ischemia and after a 90 -min reperfusion period, the PI ( $1.0 \mu \mathrm{mol} /$ liter $)$ was continuously superfused and the number of PIpositive nuclei was counted at the end of this protocol. The relationship between initial $\Delta \psi$ and PI number in each muscle fiber was assessed.

Force development. To measure active force development, the muscle was fixed at its distal attachment to a horseshoe-shaped rigid support (total weight 0.35 grams) connected by fine wire to a force transducer (0-100 grams, full scale deflection). Maximum tetanic force was measured as an index of the functional viability of the muscle. Specifically, using two partially insulated fine silver wire electrodes which contacted the entire width of the muscle belly at proximal and distal locations, the muscle was stimulated at 1.5 times the voltage necessary to achieve a maximum isometric contraction at frequencies of $10,20,40,80$, and $160 \mathrm{~Hz}$. The duration of each stimulus was $0.5 \mathrm{~ms}$. Typically, the spinotrapezius muscle elicits a fused tetanic contraction at $40-80 \mathrm{~Hz}$ (19). Baseline measurements were made with the muscle stretched to $\mathrm{L}_{0}$ (length at which maximum active tension is obtained), which was achieved by adding a preload of 2-4 grams. Ischemia was induced for $60 \mathrm{~min}$ by compressing the proximal portion of the muscle using a micromanipulator. Flow cessation in the microvasculature was confirmed by direct observation. This protocol avoids systemic complications which accompany systemic hypotensive models of ischemia. PI micrographs were digitally recorded and the muscle force was measured before ischemia and at $0,30,60$, and $90 \mathrm{~min}$ after reperfusion. These times are subsequently cited as total time from the initial control experiments (i.e., $60,90,120$, and $150 \mathrm{~min}$ ). The degree of cell injury at selected intervals was monitored by counting the mean number of PIpositive nuclei within six to seven different fields at each time point. The number of PI-positive nuclei was normalized in relation to the total number of PI-positive nuclei which were determined after 10-15 min topical application of $100 \%$ ethanol.

DMTU and SOD conditions. In separate experiments, the muscles were continuously superfused with 1,3-dimethyl-2-thiourea (DMTU, 2 $\mathrm{mM}$; Aldrich Chemical Company Inc., Milwaukee, WI) during the entire experimental protocol (150 $\mathrm{min}$ ). DMTU is highly diffusible (molecular weight: 104), scavenges the hydroxyl radical as well as hydrogen peroxide (20-22), and attenuates oxygen radical formation in $\operatorname{I} R(23,24)$. DMTU has a significant scavenging effect at a concentration of about $2 \mathrm{mM}$, and because of its diffusibility is believed to have both intracellular and extracellular effects $(20,21)$. Since a detailed pharmacokinetic study for DMTU has not been reported, we chose continuous topical application as this manner of delivery is most likely to achieve an optimal delivery to the tissues. Both the maximum tetanic force and number of PI-positive nuclei were measured under DMTU treatment.

To explore the extracellular role of superoxide anion, $\mathrm{Cu}, \mathrm{Zn}$-superoxide dismutase (Sigma Chemical Co.) was injected intravenously 30 min before the application of ischemia $(36,000 \mathrm{U} / \mathrm{kg})$ with an additional 


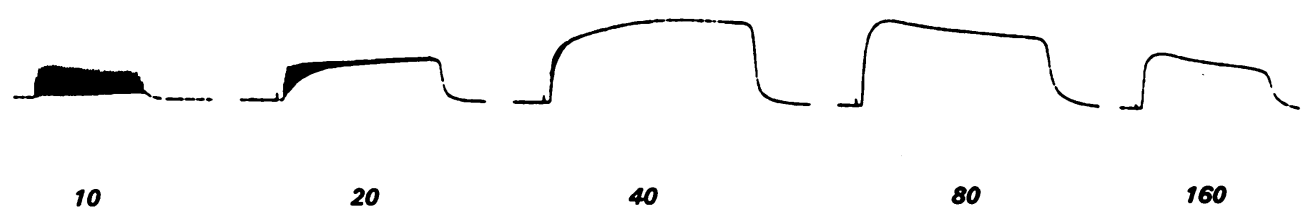

STIMULUS FREQUENCY (Hz)

Supramaxima/ voltage

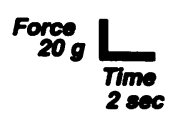

Figure 1. A representative profile of the stimulated tetanic force record. $(10,20,40,80$, and $160 \mathrm{~Hz}$ ).

intravenous dose before reperfusion $(12,000 \mathrm{U} / \mathrm{kg})$. In contrast to DMTU, SOD is a large molecule (molecular weight: 15,100 as estimated by gel filtration [25]) which does not readily gain access to the intracellular compartment $(26,27)$ and remains in the extravascular space and lymphatics (28). Thus, any scavenging effect of SOD will likely be restricted to the extracellular space. The number of PI-positive nuclei were counted. The dose of SOD was selected on the basis of pharmacokinetic studies by Korthuis et al. (28).

Statistical analysis. Statistical significance of the differences among groups was determined by two-way layout analysis of variance and Scheffe-type multiple comparison test. All values are expressed as means \pm SE. Differences were accepted as significant at $P<0.05$.

\section{Results}

During each experiment, mean arterial blood pressure remained stable at between 100 and $125 \mathrm{mmHg}$. An important index of the quality of the spinotrapezius surgical preparation and thus the suitability and viability of the muscle for subsequent study was the number of PI-positive nuclei observed at time 0 . Three muscles were rejected on this basis and none of the muscles for which data are reported had $>1$ PI-positive nucleus per field. Thus, by this criterion, all muscles were in excellent viable condition before experimental perturbation. The number of PIpositive nuclei, as determined after the application of absolute ethanol, was $650 \pm 246$ (number $/ \mathrm{mm}^{2}$, mean values $\pm \mathrm{SD}$ ).

A typical pattern of force development at different stimulation frequencies under control conditions is presented in Fig. 1. Tetanic fusion was typically achieved between 40 and $80 \mathrm{~Hz}$. A representative time course of the effect of $60 \mathrm{~min}$ ischemia followed by $90 \mathrm{~min}$ reperfusion is depicted in Fig. 2. Whilst tension development at the end of the ischemia period $(60 \mathrm{~min})$ was on average $75 \%$ of the control value, severe force reduction followed the reperfusion to levels as low as $50-60 \%$ of initial force development.

The time course of the maximum tetanic force (normalized to $100 \%$ at time 0 ) is presented in Fig. 3, top panel. There was some reduction of force generation beyond $90 \mathrm{~min}$ under control conditions. This is most likely due to surgery necessary to prepare the muscle, after which small regions of the muscle may receive inadequate blood flow. In contrast to the relatively modest decrease during ischemia, immediately after reperfusion (i.e., during the interval from 60 to $90 \mathrm{~min}$ ), the rate at which force declined was greatly accelerated. At the same time, there was a significant rise in the number of PI-positive nuclei which mirrored the decline in force (Fig. 3, bottom). Whereas the number of PI-positive nuclei did increase during the ischemic period, reperfusion induced an explosive increase within the first $30 \mathrm{~min}$. Indeed, a strong temporal correlation between the number of PI-positive nuclei and decreasing force was observed in all muscles subjected to the control and I/R protocols ( $r$ $=0.728, P<0.01$, Fig. $4 a$ ). It was interesting that the fractional reduction of maximum tetanic force tended to exceed the fraction of PI-positive nuclei (Fig. $4 a$ ).

Those muscles that were topically treated with DMTU appeared to be largely protected from the effects of the I/R protocol. Thus, both the maximum tetanic tension and the number of PI-positive nuclei remained close to those found in control muscles (Fig. 2, left and Fig. 3). The DMTU-treated muscles also exhibited a significant correlation between the reduction in force and number of PI-positive nuclei during I/R (Fig. $4 b$ ).

There was a significant inverse relationship between the initial mitochondrial function of each muscle fiber and the number of PI-positive nuclei after the entire I/R protocol (i.e., at 150 min; Fig. 5). This suggests that those fibers with either a lower initial mitochondrial volume or function were more susceptible to cell death after I/R.

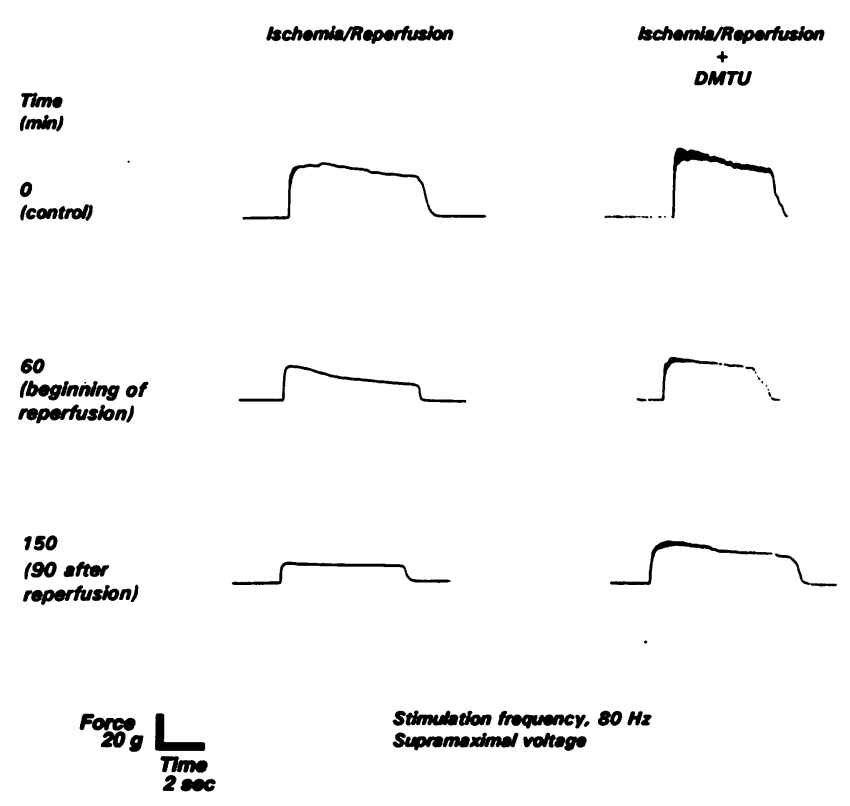

Figure 2. A representative time course profile of the changes of maximum tetanic force stimulated at $80 \mathrm{~Hz}$ in line with the protocol of ischemia-reperfusion. I/R: 1-h ischemia and after 90-min reperfusion (left), I/R + DMTU: 1-h ischemia and after 90-min reperfusion with dimethylthiourea ( $2 \mathrm{mM}$ ) treatment (right). $0 \mathrm{~min}$ : before the ischemia, $60 \mathrm{~min}$ : after 1-h ischemia, $150 \mathrm{~min}$ : after 1-h ischemia and following 90-min reperfusion. 

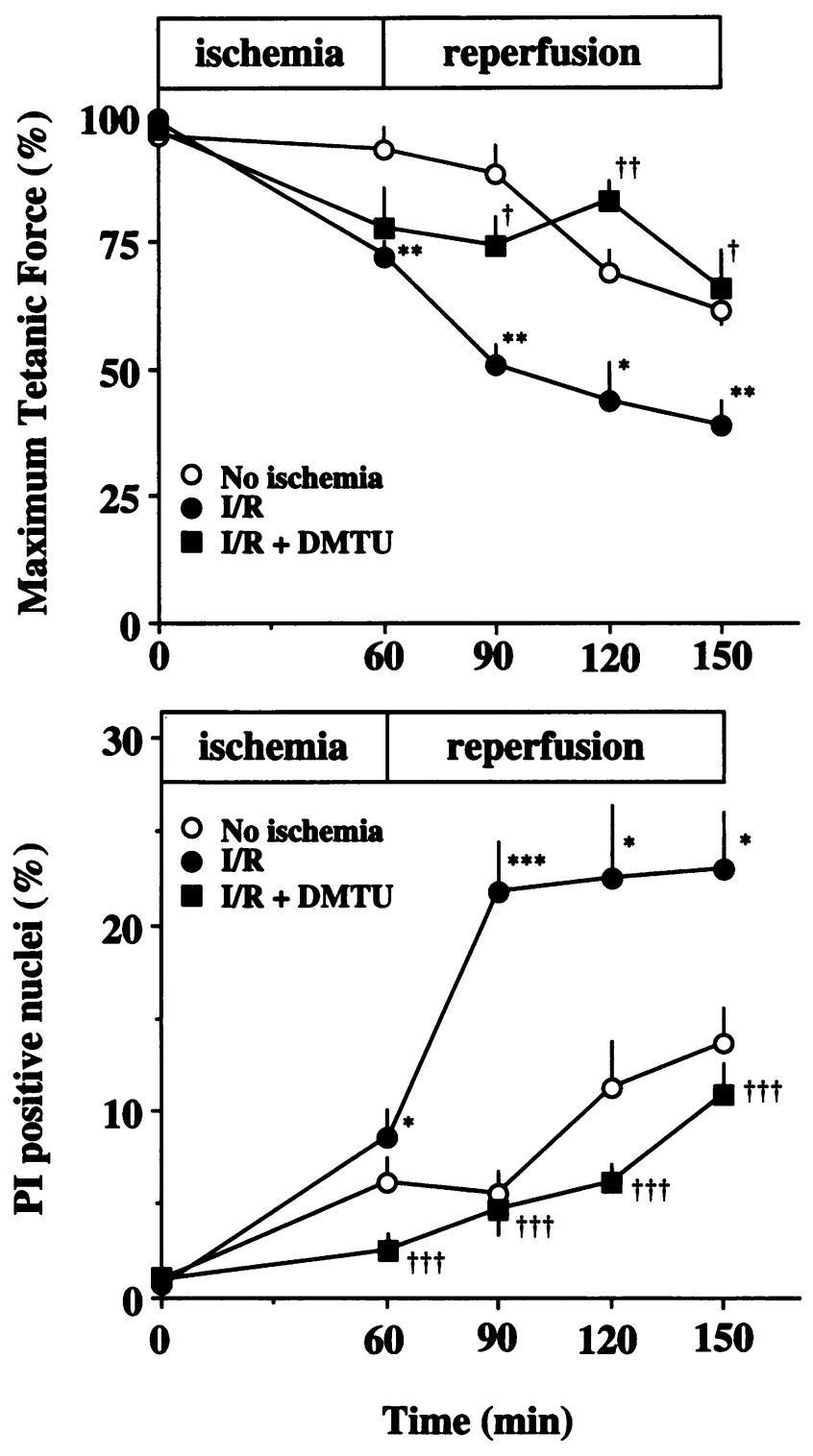

Figure 3. The temporal sequence of the maximum tetanic force (top) and the number of propidium iodide positive nuclei (\%) (bottom) among three groups (no ischemia, 60-min ischemia, and 90-min reperfusion, 60 min-ischemia and 90 -min reperfusion with DMTU treatment). I/R: 1-h ischemia and after 90-min reperfusion, I/R + DMTU: 1-h ischemia and after 90 -min reperfusion with dimethylthiourea ( $2 \mathrm{mM}$ ) treatment. Mean $\pm S E$ are shown. Four animals were examined in each group. ${ }^{*} P<0.05$ as compared with the group of no ischemia. ${ }^{* *} P$ $<0.01$ as compared with the group of no ischemia. $* * * P<0.001$ as compared with the group of no ischemia. ${ }^{\dagger}<0.05$ as compared with the group of ischemia-reperfusion. ${ }^{++}<0.01$ as compared with the group of ischemia-reperfusion. ${ }^{\dagger+\dagger}<0.001$ as compared with the group of ischemia-reperfusion.

Muscles treated with SOD during I/R showed a significantly lower number of PI-positive cells (30-min reperfusion: $4.92 \pm 1.29 \%, 60$-min reperfusion: $5.62 \pm 1.11,90$-min reperfusion: $7.42 \pm 1.26 \%, n=3$ ) compared with nontreated muscles under the $\mathrm{I} / \mathrm{R}$ protocol $(P<0.05)$. There was no significant difference in PI staining after I/R between the DMTU-treated and the SOD-treated group, suggesting that the extracellular
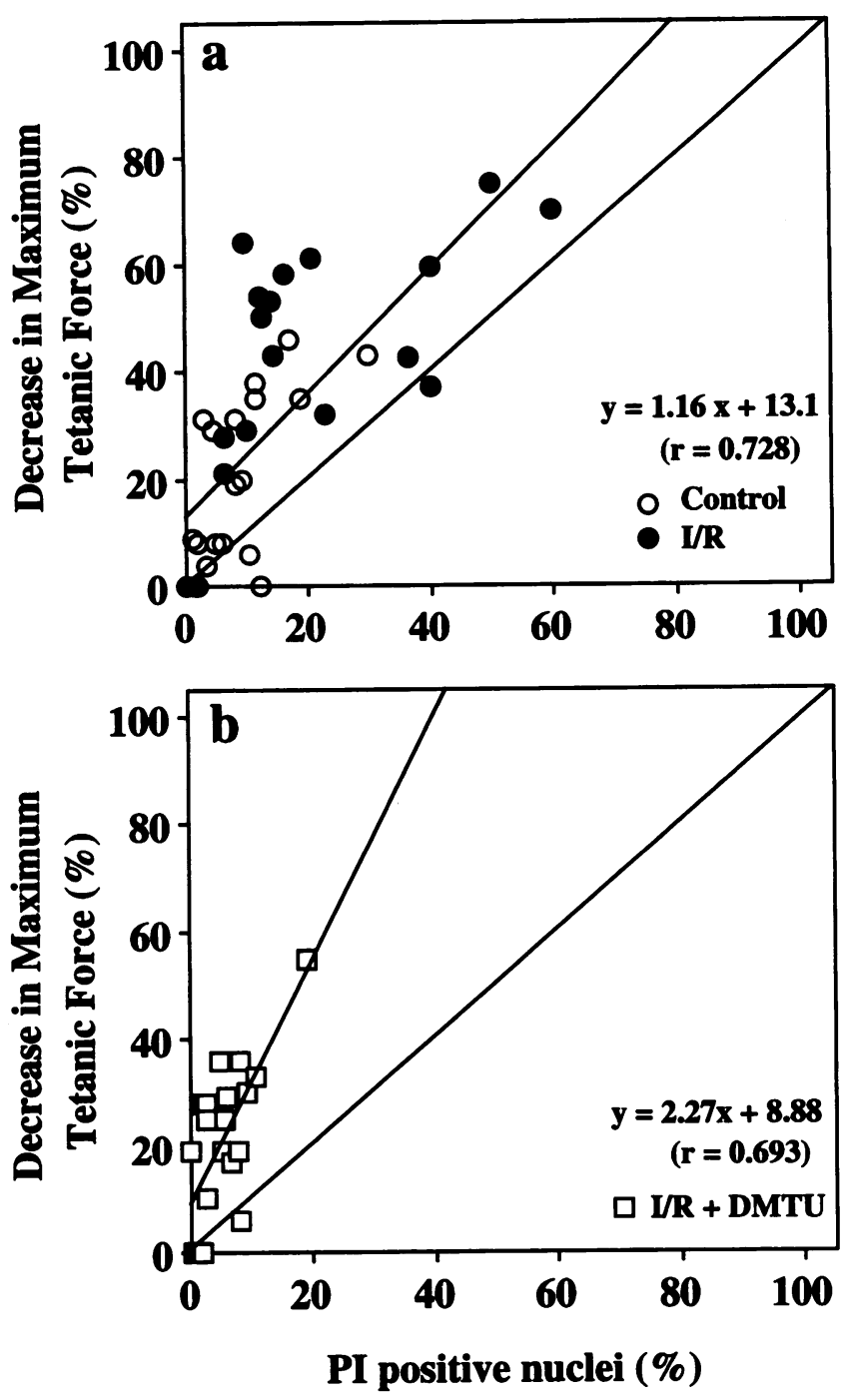

Figure 4. The correlation between the rise in number of propidium iodide positive nuclei $(\%)$ and the decrease in maximum tetanic force $(\%)$ under control and ischemia-reperfusion conditions $(a)$ or under ischemia-reperfusion treated with dimethylthiourea (DMTU, $2 \mathrm{mM}$ ) (b). As reference, the lines of identity are shown. There is a significant linear correlation between the two parameters $(a)(r=0.728,[\%$ decrease in Tension $]=1.16 \times[\%$ PI-positive nuclei $]+13.1,(b)$ $(r=0.693$, [\% decrease in tension] $=2.27 \times[\%$ PI-positive nuclei] $+8.88)$.

damaging mechanism by oxygen radicals predominates in this muscle.

\section{Discussion}

The current observations demonstrate that the major portion of the tetanic force degeneration in skeletal muscle occurs during reperfusion, hand in hand with an increase in myocyte death and deterioration of mitochondrial function. Cell death during ischemia is minimal (9) and good mitochondrial function is sustained. The extent of the damage to pericapillary nuclei is not uniform among the various fiber types of the postischemic rat spinotrapezius muscle. Fast glycolytic fibers (type IIB fibers) show cell death during early reperfusion with only mini- 


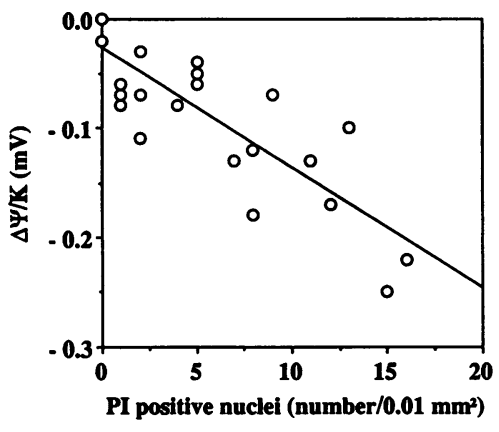

Figure 5. The correlation between the number of PI-positive nuclei $90 \mathrm{~min}$ after ischemia-reperfusion protocol (number/ $0.01 \mathrm{~mm}^{2}$ ) and the net initial mitochondrial inner membrane potential $(\Delta \psi / \mathrm{K})$. There is a significant linear inverse correlation between the two parameters $(r$ $=0.828,[\Delta \psi / \mathrm{K}$

$(\mathrm{mV})]=-0.01 \times\left[\right.$ PI-positive nuclei $\left(\right.$ number $\left.\left.\left./ 0.01 \mathrm{~mm}^{2}\right)\right]-0.03\right)$. The number of muscle fibers examined was 25 .

mal involvement of other fiber types. The heterogeneity of myocyte susceptibility among the different fibers is likely the consequence of fiber-type specific oxygen radical generation and scavenging mechanisms (9). The presence and effectiveness of antioxidant enzymes such as SOD and glutathione peroxidase is more evident in oxidative fibers when compared with glycolytic fibers $(29,30)$.

The relative susceptibility of fast glycolytic (type IIB) fibers to I/R most likely reflects an overabundance of oxygen radicals in relation to endogenous scavenging enzymes. Under physiologic conditions of oxidative stress, it can be envisaged that the higher SOD, catalase (CAT), and glutathione peroxidase (GPX) activities of type I ( slow oxidative) and IIA (fast oxidative glycolytic) fibers (31) would compensate for the production of oxygen radicals during reperfusion and prevent tissue damage. Accordingly, a lower SOD, CAT, and GPX activity in type IIB (fast glycolytic) fibers reflects the lower oxidative function and thus production of oxygen radicals in these fibers. However, under I/R conditions it is conceivable that an excess of oxygen caused by reactive hyperemia in combination with a relative surplus of available capillaries $(32,33)$ in relation to mitochondrial oxygen utilization might raise intracellular $\mathrm{PO}_{2}$ and promote a high oxygen radical production. $\mathrm{O}_{2}^{-}$produced by intracellular mechanisms moves through cell membranes via anion channels (34) and may also target cells from extracellular sites. In this context, type IIB fibers would be less able to ward off damage. Both membrane permeable DMTU and impermeable SOD significantly attenuate cell death after I/R without significant difference in magnitude of protection between these two interventions, suggesting that the main pathway of oxygen radicals in this model might be via the extracellular space.

The idea that the type IIB fibers are selectively vulnerable to ischemic injury has been proposed previously. Jennische demonstrated ischemia-induced injury to glycogen-depleted skeletal muscle, which was specific to the type IIB fibers (35). During ischemia, a proportionally large number of the type IIB fibers degenerate in comparison with type I or IIA fibers (7). However, the skeletal muscle fiber type may not consistently serve as a predictive index for the sensitivity to $I / R$-induced injury (36). The compartmental location of muscle fibers in modulating vulnerability to postischemic damage may also play a contributory role (36). The present study supports the notion that the initial mitochondrial function (or volume) as reflected by the net mitochondrial inner membrane potential ( $\Delta \psi$; computed by relative Rh123 fluorescence intensity) enables one to predict the resistance or susceptibility of a given myocyte to injury during I/R (Fig. 5).

The effects of $I / R$ on force generation in skeletal muscle are characterized by a different time course than encountered in heart muscle. Heart muscle becomes akinetic immediately after ischemia, but may recover to almost full function immediately upon reperfusion, subsequently progressing to failure during the reperfusion period. Thus, cells in both cardiac and skeletal muscle appear to be viable at the end of 1-h ischemia and loss of function occurs predominantly upon reperfusion $(9,37)$. In both cases, it is likely that oxygen radical formation during reperfusion precipitates the compromised mitochondrial function and cell death. The period of 1-h ischemia, although quite damaging in rats is too short to induce significant injury and significant necrosis in higher species, including humans. The reason for this discrepancy may be due to the difference in basal metabolic rate (BMR). The value of BMR in rats (29.7 $\mathrm{ml} \mathrm{O}_{2} / \mathrm{kg}$ per $\mathrm{min}$ ) (38) is $\sim 8$ times higher than that in humans (3.6 $\mathrm{ml} \mathrm{O}_{2} / \mathrm{kg}$ per min) (39).

On average, type I (oxidative) fibers contain 650 nuclei per $\mathrm{mm}^{2}$ compared with 570 nuclei per $\mathrm{mm}^{2}$ for type IIB (glycolytic) fibers (40). Since the spinotrapezius muscle is a mixture of these fibers, the average number of nuclei present in the tissue should fall within this range, i.e., 600 nuclei per $\mathrm{mm}^{2}$ of tissue. In the present study, the number of PI-positive nuclei, as determined after the application of absolute ethanol, was slightly higher ( 650 nuclei per $\mathrm{mm}^{2}$ of tissue). This small discrepancy may potentially be ascribed to our inclusion of nonmuscle cells in the PI count, such as endothelial cells and fibroblasts. The maximum cell injury after $I / R$, as indicated by the number of PI-stained nuclei, was 196 per $\mathrm{mm}^{2}$; in other words, $\sim 30 \%$ of the cell population in spinotrapezius muscle was damaged in response to the ischemia-reperfusion challenge. This value corresponds broadly to the proportion of type IIB fibers and is consistent with the evidence that this is the most sensitive fiber type to $I / R$ (9). The percent ratio of crosssectional area for type I ( slow oxidative), type IIA (fast oxidative), and type IIB (fast glycolytic) fibers in rat spinotrapezius muscle is $25: 27: 48 \%$ as calculated from previous measurements (19). Bodine et al. (41) found that the maximal tensile stress produced by fast fibers was $36 \%$ higher than that produced by slow fibers. Other authors have reported that the disparity of tensile stress between fast glycolytic and slow oxidative fibers was even greater. For example, Dum et al. (42) reported that the tensile stress was sixfold greater in fast twitch fibers compared with their slow twitch counterparts. Thus type IIB (fast glycolytic) fibers may be responsible for as much as $50-60 \%$ of the total tension development of the spinotrapezius muscle with type I and IIA fibers each contributing a substantially lower proportion of the total force. On the basis of these considerations, it is plausible that dysfunction of the entire type IIB fiber population could precipitate a decrease of force by $50-60 \%$. This estimation is compatible with the present investigation in which maximum tetanic force was reduced to $\sim 40 \%$ of control after I/R (Fig. 3, top). However, we have no direct evidence that damage to type I and IIA fibers was not involved in the force reduction after I/R. Thus, the potential contribution of damage to types I and IIA fibers in this investigation cannot be discounted.

In conclusion, this investigation demonstrates that structural indices of I/R-induced muscle injury reflect directly the impaired contractile function. The oxygen radical scavengers, 
DMTU and SOD, substantially ameliorate the deficits arising in the postischemic period suggesting a deterministic role of oxygen radicals in $I / R$ injury and the associated muscle dysfunction.

\section{Acknowledgments}

This work was completed during the tenure of a research fellowship for Dr. Hidekazu Suzuki from the American Heart Association, California Affiliate.

This work was supported by National Institute of Health grants HL10881, HL-43026, and HL-50306.

\section{References}

1. Makitie, J. 1977. Microvasculature of rat striated muscle after temporary ischemia. Acta Neuropathol. 37:247-253.

2. Korthuis, R. J., D. N. Granger, M. I. Townsley, and A. B. Taylor. 1985 The role of oxygen derived free radicals in ischemia induced increases in canine skeletal muscle microvascular permeability. Circ. Res. 57:599-609.

3. Korthuis, R. J., J. K. Smith, and D. L. Carden. 1989. Hypoxic reperfusion attenuates postischemic microvascular injury. Am. J. Physiol. 256:H315-H319.

4. Harris, K., P. M. Walker, D. A. G. Mickle, R. Harding, R. Gately, G. J. Wilson, B. Kuzon, N. McKee, and A. D. Romaschin. 1986. Metabolic response of skeletal muscle to ischemia. Am. J. Physiol. 250:H213-H220.

5. Makitie, J., and H. Teravainen. 1978. Ultrastructure of striated muscle of the rat after temporary ischemia. Acta Neuropathol. 37:237-245.

6. Sternbergh, W. S., and B. Adelman. 1992. The temporal relationship between endothelial cell dysfunction and skeletal muscle damage after ischemia and reperfusion. J. Vasc. Surg. 16:30-39.

7. Caiozzo, V. J., V. O. Gardner, K. Starr, H. Najarian, and C. A. Prietto. 1990. Fast fibers are more susceptible to ischemia. In Transaction of the 36th Annual Meeting of the Orthopaedic Research Society 1990. vol. 15. The Orthopaedic Research Society. Editor Park Ridge. 145.

8. Lieber, R. L., R. A. Pedowitz, J. Friden, and D. H. Gershuni. 1992. Decreased muscle speed, strength and fatigability following two hours of tourniquetinduced ischemia. Scand. J. Plast. Reconstr. Surg. Hand Surg. 26:127-132.

9. Suematsu, M., F. A. DeLano, D. C. Poole, R. L. Engler, M. Miyasaka, B. W. Zweifach, and G. W. Schmid-Schönbein. 1994. Spatial and temporal correlation between leukocyte behavior and cell injury in postischemic rat skeletal muscle microcirculation. Lab. Invest. 70:684-695.

10. Gardner, V. O., V. J. Caiozzo, S. T. Long, J. Stoffel, W. C. McMaster C. A. Prietto. 1984. Contractile properties of slow and fast muscle following tourniquet ischemia. Am. J. Sports Med. 12:417-423.

11. Pedowitz, R. A., J. Friden, and L.-E. Thornell. 1992. Skeletal muscle injury induced by a pneumatic tourniquet: an enzyme- and immunochemical study in rabbits. J. Surg. Res. 52:243-250.

12. Adembri, C., L. L. Domenici, L. Formigli, S. Brunelleschi, E. Ferrari, and G. P. Novelli. 1994. Ischemia-reperfusion of human skeletal muscle during aortoiliac surgery: effects of acetylcarnitine. Histol. Histopath. 9:683-690.

13. Suzuki, H., M. Suematsu, H. Ishii, S. Kato, H. Miki, M. Mori, Y. Ishimura T. Nishino, and M. Tsuchiya. 1994. Prostaglandin $E_{1}$ abrogates early reductive stress and zone-specific oxidative injury in hypoperfused rat liver. J. Clin. Invest. 93:155-164.

14. Gray, S. D. 1973. Rat spinotrapezius muscle preparation for microscopic observation of the terminal vascular bed. Microvasc. Res. 5:395-400.

15. Mazzoni, M. C., T. C. Skalak, and G. W. Schmid-Schönbein. 1990. Effects of skeletal muscle fiber deformation on lymphatic volumes. Am. J. Physiol. 259:H1860-H1868.

16. Suematsu, M., H. Suzuki, H. Ishii, S. Kato, H. Hamamatsu, S. Miura, and M. Tsuchiya. 1992. Topographic dissociation between mitochondrial dysfunction and cell death during low-flow hypoxia in perfused rat liver. Lab. Invest. 67:434442 .

17. Lemasters, J. J., J. DiGiuseppi, A.-L. Nieminen, B. Herman. 1987. Blebbing, free $\mathrm{Ca}^{2+}$ and mitochondrial membrane potential preceding cell death in hepatocytes. Nature (Lond.). 325:78-81.

18. Emanus, R. K., R. Grunwald, and J. J. Lemasters. 1986. Rhodamine 123 as a probe of transmembrane potential in isolated rat liver mitochondria: spectral and metabolic properties. Biochim. Biophys. Acta. 850:436-448.

19. Taylor, K., and T. N. Calvey. 1977. Histochemical characteristics and contractile properties of the spinotrapezius muscle in the rat and the mouse. $J$. Anat. 123:67-76.

20. Fox, R. B. 1984. Prevention of granulocyte-mediated oxidant lung injury in rats by a hydroxyl radical scavenger, dimethylthiourea. J. Clin. Invest. $74: 1456-1464$.

21. Kaufman, Jr., R. P., J. M. Klausner, H. Anner, H. Feingold, L. Kobzik, C. R. Valeri, D. Shepro, and H. B. Hechtman. 1988. Inhibition of thromboxane synthesis by free radical scavengers. J. Trauma. 28:458-464.

22. Jackson, J. H., C. W. White, N. B. Parker, J. W. Ryan, and J. E. Repine. 1985. Dimethylthiourea consumption reflects $\mathrm{H}_{2} \mathrm{O}_{2}$ concentrations and severity of acute lung injury. J. Appl. Physiol. 59:1995-1998.

23. Suematsu, M., G. W. Schmid-Schönbein, R. H. Chavez-Chavez, T. T. Yee, T. Tamatani, M. Miyasaka, F. A. DeLano, and B. W. Zweifach. 1993. In vivo visualization of oxidative changes in microvessels during neutrophil activation. Am. J. Physiol. 264:H881-H891.

24. Suzuki, H., A. Swei, B. W. Zweifach, and G. W. Schmid-Schönbein. 1995. In vivo evidence for microvascular oxidative stress in spontaneously hypertensive rats: hydroethidine microfluorography. Hypertension (Dallas). 25:10831089.

25. Tibell, L., K. Hjalmarsson, T. Edlund, G. Skogman, E. Engstrom, and S. L. Marklund. 1987. Expression of human extracellular superoxide dismutase in Chinese hamster ovary cells and characterization of the product. Proc. Natl. Acad. Sci. USA. 84:6634-6638.

26. Paky, A., J. R. Michael, T. M. Burke-Wolin, M. S. Wolin, and G. H. Gurtner. 1993. Endogenous production of superoxide by rabbit lungs: effects of hypoxia or metabolic inhibitors. J. Appl. Physiol. 74:2868-2874.

27. Akimitsu, T., D. C. Gute, and R. J. Korthuis. 1995. Leukocyte adhesion induced by inhibition of nitric oxide production in skeletal muscle. J. Appl. Physiol. 78:1725-1732.

28. Korthuis, R. J., P. Kubes, P. Tso, M. Perry, and D. N. Granger. 1991. Transport kinetics for superoxide dismutase and catalase between plasma and intestinal fluid in the rat small intestine. Free Radical Biol. \& Med. 11:293-298.

29. Sexton, W. L., R. J. Korthuis, and M. H. Laughlin. 1990. Microvascular injury after ischemia and reperfusion in skeletal muscle of exercise-trained rats. J. Appl. Physiol. 68:2329-2336.

30. Apple, F. S., J. E. Hyde, A. M. Ingersoll-Stroubos, and A. Theologides. 1991. Geographic distribution of xanthine oxidase, free radical scavengers, creatinine kinase, and lactate dehydrogenase enzyme systems in rat heart and skeletal muscle. Am. J. Anat. 192:319-323.

31. Laughlin, M. H., T. Simpson, W. L. Sexton, O. R. Brown, J. K. Smith, and R. J. Korthuis. 1990. Skeletal muscle oxidative capacity, antioxidant enzymes, and exercise training. J. Appl. Physiol. 68:2337-2343.

32. Hudlicka, O., H. Hoppeler, and E. Uhlmann. 1987. Relationship between the size of the capillary bed and oxidative capacity in various cat skeletal muscles. Pflügers Arch. 410:369-375.

33. Gray, S. D., and E. M. Renkin. 1977. Microvascular supply in relation to fiber metabolic type in mixed skeletal muscles of rabbits. Microvasc. Res. 16:406425 .

34. Lynch, R. E., and J. Fridovich. 1978. Permeation of the erythrocyte stroma by superoxide radical. J. Biol. Chem. 253:1838-1845.

35. Jennische, E. 1985. Ischemia-induced injury in glycogen-depleted skeletal muscle. Selective vulnerability of FG fibers. Acta Physiol. Scand. 125:727-734. 36. Sternbergh, III, W. C., and B. Adelman. 1992. Skeletal muscle fiber type does not predict sensitivity to postischemic damage. J. Surg. Res. 53:535-541.

37. Bolli, R. 1991. Oxygen-derived free radicals and myocardial reperfusion njury: an overview. Cardiovasc. Drugs Ther. 5:249-268.

38. Brooks, G. A., and T. P. White. 1978. Determination of metabolic and heart rate responses of rats to treadmill exercise. J. Appl. Physiol. Respir. Environ. Exercise Physiol. 45:1009-1015.

39. McArdle, W. D., F. I. Katch, and V. L. Katch. 1991. Exercise Physiology: Energy, nutrition and human performance. Lea \& Febiger, Philadelphia. 853 pp. 40. Atherton, G. W., and N. T. James. 1980. Stereological analysis of the number of nuclei in skeletal muscle fibers. Acta Anat. 107:236-240.

41. Bodine, S. C., R. R. Roy, E. Eldred, and R. Edgerton. 1987. Maximal force as a function of anatomical features of motor units in the cat tibialis anterior. J. Neurophysiol. 57:1730-1745.

42. Dum, R. P., R. E. Burke, M. J. O’Donovan, J. Toop, and J. A. Hodgson. 1982. Motor-unit organization in flexor digitorum longus muscle of the cat. $J$. Neurophysiol. 47:1108-1125. 